\title{
Shift of Shapiro Step in High Critical Temperature Superconductors
}

\author{
Kirill Kulikov ${ }^{1,2, \star}$, Yury Shukrinov ${ }^{1,2}$, Majed Nashaat ${ }^{1,3}$, and Akinobu Irie ${ }^{4}$ \\ ${ }^{1}$ BLTP, JINR, Dubna, Moscow Region, 141980, Russia \\ ${ }^{2}$ State University of "Dubna", Dubna, Moscow region, 141980 Russia \\ ${ }^{3}$ Department of Physics, Cairo University, Cairo, Egypt \\ ${ }^{4}$ Department of Electrical and Electronic Systems Engineering, Utsunomiya University, Japan
}

\begin{abstract}
The influence of the charge imbalance effect on a system of intrinsic Josephson junctions of high temperature superconductors under external electromagnetic radiation is investigated. We find that the charge imbalance is responsible for the slope of the Shapiro step in the IV-characteristic. The nonperiodic boundary conditions shift the Shapiro step from the canonical position which is determined by the frequency of the external radiation. We also find how the system parameters influence the shift of the Shapiro step.
\end{abstract}

\section{Introduction}

The phase dynamics of the layered superconducting materials have attracted a great interest because of the rich and interesting physics on one side and application perspectives on the other side $[1,2]$. In particular, the nonequilibrium effects created by stationary current injection in high- $T_{c}$ materials have been studied very intensively during the last two decades [1-7]. However, the charge imbalance in the systematic perturbation theory was considered only indirectly as far as it is induced by fluctuations of the scalar potential $[1,2,5]$. In Ref. [8], it is taken into account as an independent degree of freedom and therefore the results are different from those of earlier treatments. In addition, due to the fact that the charge is not screened in the superconducting layers the system forms intrinsic Josephson junctions (IJJ) $[9,10]$. Such system cannot be at equilibrium at any value of the electrical current. The influence of the charge coupling on the Josephson plasma oscillations was studied in [6,9].

During the last few years, two theoretical models are widely used to describe the IJJ: the capacitively coupled Josephson junctions (CCJJ) model and the charge imbalance (CIB) model. In the CCJJ model a non-vanishing generalized scalar potential appears due to the breaking of the charge neutrality, but in CIB model it is related to the quasiparticle charge imbalance. Actually, the relaxation length of the charge imbalance in the layered system could be much larger than any other characteristic lengths. Therefore, both effects could exist in HTSC simultaneously because the thickness of the superconducting layers is smaller than the Debye length and thus obviously less than the relaxation length of the charge imbalance. In the present paper we study the nonequilibrium effects created by current injection in a stack of IJJ under external electromagnetic radiation.

\footnotetext{
${ }^{\star}$ e-mail: kulikov@theor.jinr.ru
} 


\section{Model}

A system of $N+1$ superconducting layers (S-layers) presented in Fig. 1 is characterized by the order parameter $\Delta_{l}(t)=|\Delta| \exp \left(i \theta_{l}(t)\right)$ and time-dependent phase $\theta_{l}(t)$. The thickness of the $S$-layer is

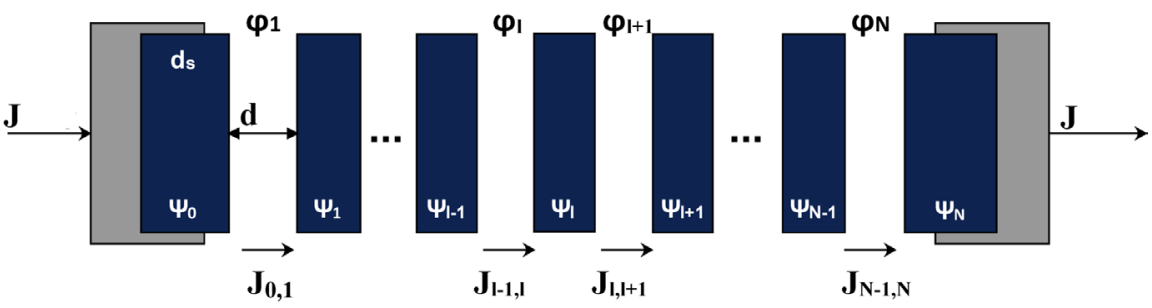

Figure 1. Layered system of $N+1$ superconducting layers forms a stack of Josephson junctions. Since the 0-th and $N$-th layers are in contact with normal metal, their thicknesses $d_{s}^{0}$ and $d_{s}^{N}$ are different from the thickness of the other S-layers $d_{s}$ inside of the stack due to the proximity effect.

comparable with the Debay screening $r_{D}$ length that leads to the generalized Josephson relation [11]. The total current density $J_{l}$ through each S-layer is given as a sum of displacement, superconducting, quasiparticle, diffusion and nonequilibrium terms. Those equations together with the kinetic equations for the nonequilibrium potential $\Psi_{l}(t)$ describe the physics of IJJs in HTSC. In the dimensionless form the system of equations is

$$
\begin{aligned}
\dot{v}_{l} & =\left[I-\sin \varphi_{l}-\beta \dot{\varphi}_{l}+A \sin \omega \tau+I_{\text {noise }}+\psi_{l}-\psi_{l-1}\right], \\
\dot{\varphi}_{1} & =v_{1}-\alpha\left(v_{2}-(1+\gamma) v_{1}\right)+\frac{\psi_{1}-\psi_{0}}{\beta}, \\
\dot{\varphi}_{l} & =(1+2 \alpha) v_{l}-\alpha\left(v_{l-1}+v_{l+1}\right)+\frac{\psi_{l}-\psi_{l-1}}{\beta}, \\
\dot{\varphi}_{N} & =v_{N}-\alpha\left(v_{N-1}-(1+\gamma) v_{N}\right)+\frac{\psi_{N}-\psi_{N-1}}{\beta}, \\
\zeta_{0} \dot{\psi}_{0} & =\eta_{0}\left(I+A \sin \omega \tau-\beta \dot{\varphi}_{0,1}+\psi_{1}-\psi_{0}\right)-\psi_{0}, \\
\zeta_{l} \dot{\psi}_{l} & =\eta_{l}\left(\beta\left[\dot{\varphi}_{l-1, l}-\dot{\varphi}_{l, l+1}\right]+\psi_{1-1}+\psi_{l+1}-2 \psi_{l}\right)-\psi_{l}, \\
\zeta_{N} \dot{\psi}_{N} & =\eta_{N}\left(-I-A \sin \omega \tau+\beta \dot{\varphi}_{N-1, N}+\psi_{N-1}-\psi_{N}\right)-\psi_{N},
\end{aligned}
$$

where the dot shows the derivative with respect to $\tau=\omega_{p} t, v_{l}$ between the layers $l-1$ and $l, v_{l}(t) \equiv$ $v_{l, l-1}(t), \varphi_{l}(t)$ is the phase difference across the layers $l-1$ and $l, \alpha=\epsilon \epsilon_{o} / 2 e^{2} N(0) d$ is the coupling parameter, $\epsilon$ is the dielectric constant, $\epsilon_{o}$ is the vacuum permittivity, $d$ is the distance between the superconducting layers and $N(0)$ is the density of states, $I=J / J_{c}$ is the dimensionless current density, $J_{c}$ is the critical current density, $\omega_{p}=\sqrt{2 e J_{c} /(\hbar C)}$ is the plasma frequency, $C$ is the capacitance. Other dimensionless parameters are the dissipation parameter $\beta=\hbar \omega_{p} /\left(2 e R I_{c}\right), R$ is the junction resistance, the normalized quasiparticle relaxation time $\zeta_{l}=\omega_{p} \tau_{q p}$ and the nonequilibrium parameter $\eta_{l}=4 \pi r_{D}^{2} \tau_{q p} /\left(d_{s}^{l} R\right)$, where $d_{s}^{i}$ is the thickness of the S-layers, and $\tau_{q p}$ is the quasiparticle relaxation time. The parameter of the nonperiodic boundary conditions $\gamma$ is $\gamma=d_{s} / d_{s}^{0}=d_{s} / d_{s}^{N}$. The term $A \sin \omega \tau$ describes the effect of the external radiation with the amplitude $A$ and frequency $\omega$, which are normalized to $J_{c}$ and $\omega_{p}$, respectively. To reflect the experimental situation, we have added the noise $I_{\text {noise }}$ in the bias current with the amplitude $\sim 10^{-8}$ which is produced by a random number generator and its amplitude is normalized to the critical current density value $J_{c}$. 
This system of equations is solved numerically using the fourth order Runge-Kutta method. We assume that due to the proximity effect the thickness of the first and the last S-layers are larger than the middle one. Therefore, the nonequilibrium parameters depend on the parameter of boundary conditions $\gamma, \eta_{0, N}=\gamma \eta_{l}$, where $l=1,2, \ldots, N-1$. We consider the underdamped case with the McCumber parameter $\beta_{c}=25$ or $\beta=0.2$.

\section{Results}

In [11] we have shown that in the system of intrinsic Josephson junctions of high temperature superconductors under external electromagnetic radiation the charge imbalance is responsible for the slope in the Shapiro step in the IV-characteristic. The value of the slope increases with the nonequilibrium parameter. We demonstrate that the coupling between junctions leads to the distribution of the slope values along the stack. It was shown also that the nonperiodic boundary conditions shift the Shapiro step from the canonical position $V=N \omega$.

The simulated IV-characteristics of the JJs stack in the case without the charge imbalance $\eta=0$ (dashed line) and at $\eta=0.2$ (solid line) are presented in Fig. 2. The IV-curve without the charge

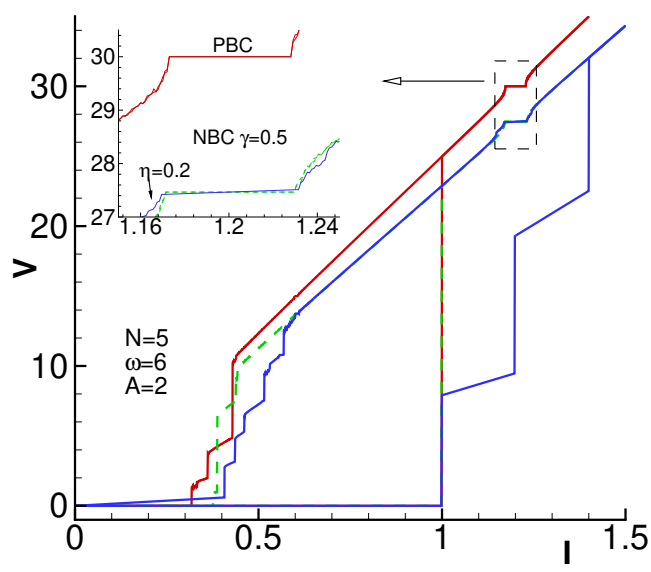

Figure 2. The IV-characteristics of JJ stacks without the charge imbalance $\eta=0$ (dashed line) and at $\eta=0.2$ (solid line). The results for the periodic boundary condition are shown for comparison. The enlarged parts of the IV-characteristics with the SS are shown in the inset.

imbalance at periodic boundary conditions is shown as well. The simulations have been made for a stack with five JJs, a coupling parameter $\alpha=0.5$ and $\gamma=0.5$. We see that the position of the SS (at periodic boundary condition (PBC)) corresponds to the canonical value of the SS voltage $V=30$ in agreement with the value of the external frequency $\omega=6$ and a number of junctions in the stack $N=5$. The nonperiodic boundary conditions with $\gamma \neq 0$ shifts the outermost branch relatively to the curve at PBC, leading to a corresponding shift of the Shapiro steps. The charge imbalance manifests itself as appearance of the slope in the Shapiro step, which is clearly demonstrated in the inset for the case $\eta=0.2$.

The influence of the coupling parameter on the shift of the Shapiro step is shown in Fig. 3(a). The increase of $\alpha$ leads to the increase of the shift value. The steps on the IV-characteristic at $\alpha=$ $0,0.2,0.6,1$ are indicated by the large dashed rectangle. The IV-characteristics at large $\alpha$ demonstrate also additional steps to those occurring on an internal branch. These Shapiro steps are evidenced inside the inset to the small dashed rectangle. 

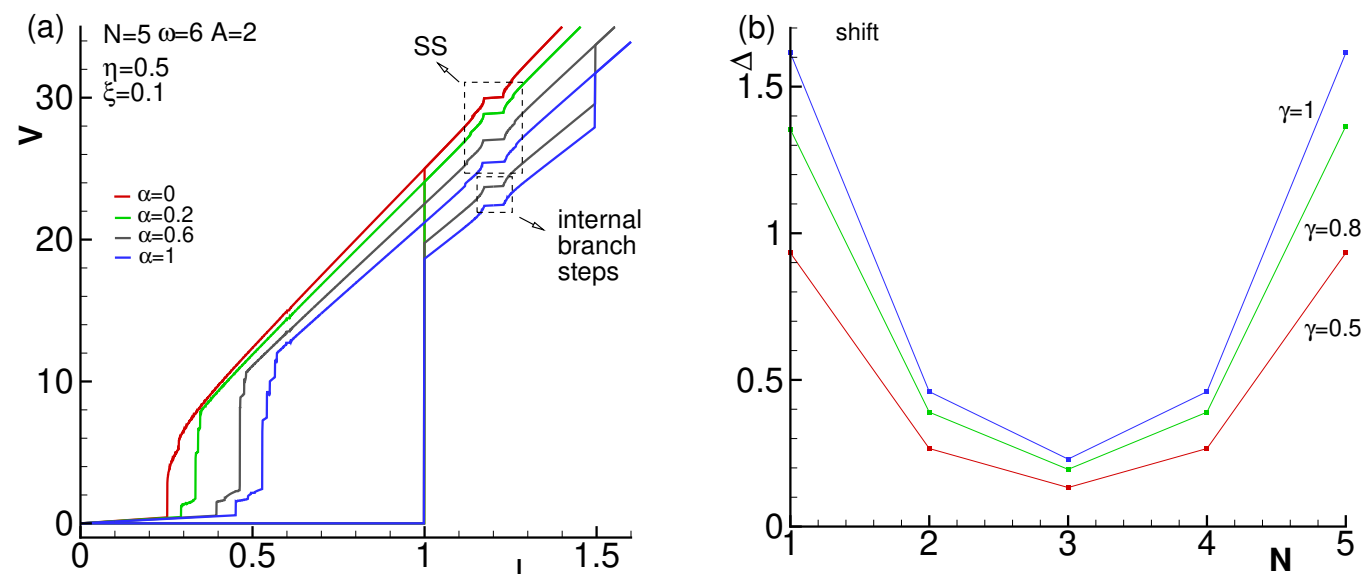

Figure 3. (a) IV-characteristics of the stack of $N=5 \mathrm{JJ}$ at four coupling parameter values $\alpha=0,0.2,0.6,1$. (b) Distribution of the SS shift along the stack of $N=5 \mathrm{JJ}$ at three parameter values $\gamma=0.5,0.8,1$.

The shift of the Shapiro step depends on the value of $\gamma$ and on the coupling parameter $\alpha$. Fig. 3(b) shows the distribution of the shift of the step along the stack with parameters $\gamma=0.5,0.8,1$. One can see that the maximum of the shift values occur at the first and last Josephson junctions. The thicknesses of the superconducting layers of those junctions are larger than the others. The distribution of the shift can be also seen in the middle layers due to the coupling between the Josephson junctions.

Thus, the Shapiro step shows a shift of its position from the canonical value $N \omega$, where $N$ is the number of junctions in the stack and $\omega$ is the frequency of the external radiation. The value of this shift depends on the boundary conditions and the coupling between the Josephson junctions. Due to the coupling, the effect of the boundary conditions is extended to the neighboring junctions.

\section{Acknowledgement}

The reported study was funded by RFBR according to the research projects 15-29-01217 and 16-5245011_ India.

\section{References}

[1] S. Artemenko and A. Kobelkov, Phys. Rev. Lett. 78, 3551 (1997)

[2] C. Preis, C. Helm, J. Keller, A. Sergeev, and R. Kleiner, in Superconducting Superlattices II: Native and Artificial (Proceedings of SPIE Volume 3480, 1998) p. 236

[3] S. E. Shafranjuk and M. Tachiki, Phys. Rev. B 59, 14087 (1999)

[4] C. Helm, C. Preis, C. Walter, and J. Keller, Phys. Rev. B 62, 6002 (2000)

[5] C. Helm, J. Keller, C. Preis, and A. Sergeev, Physica C 362, 43 (2001)

[6] C. Helm, L. N. Bulaevskii, E. M. Chudnovsky, and M. P. Maley, Phys. Rev. Lett. 89, 057003 (2002)

[7] L. N. Bulaevskii, C. Helm, A. R. Bishop, and M. P. Maley, Europhys Lett. 58, 057003 (2002)

[8] J. Keller and D. A. Ryndyk, Phys. Rev. B 71, 054507 (2005)

[9] T. Koyama and M. Tachiki, Phys. Rev. B 54, 16183 (1996)

[10] D. A. Ryndyk, Phys. Rev. Lett. 80, 3376 (1998)

[11] Yu. M. Shukrinov, M. Nashaat, K. V. Kulikov, R. Dawood, H. El Samman, and Th. M. El Sherbini, EPL 115, 20003 (2016) 